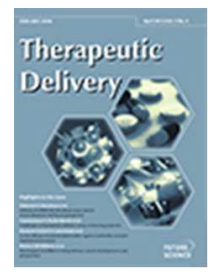

\title{
Influences of the vehicle in the spreading and release of betamethasone
}

\begin{tabular}{|r|l|}
\hline Journal: & Therapeutic Delivery \\
\hline Manuscript ID & TDE-2017-0070.R2 \\
\hline Manuscript Type: & Preliminary Communication \\
\hline Keywords: & lipid-enriched vehicle, betamethasone, topical drug \\
\hline \multicolumn{2}{|l}{} \\
\hline
\end{tabular}

\section{SCHOLARONE ${ }^{\mathrm{IM}}$ \\ Manuscripts}


1

2

3

4

5

6

7

8

9

10

11

12

13

14

15

16

17

18

19

20

21

22

23

24

25

26

27

28

29

30

31

32

33

34

35

36

37

38

39

40

41

42

43

44

45

46

47

48

49

50

51

52

53

54

55

56

57

58

59

60
1 Article

2 Influences of the vehicle in the spreading and release of betamethasone

3

4

5

6

Running title: Vehicles for bethametasone

7 Word count: 2987

8 Tables: 5

9

10

11

12

13

14

15

16

17

18

19

20

21

22

23

24 Abstract 
1 Background

2 We compared the performances of two different commercial products both based on

3 betamethasone and an antibiotic but using different pharmaceutical vehicles: a polymer and lipid-

4 enriched cream and a conventional oil in water (O/W) emulsion.

5 Methodology

6 Evaluation was conducted on a reconstructed human epidermis (RHE) model. Moreover, skin

7 barrier properties and cutaneous hydration of the solely two vehicles- were evaluated on 20

8 human healthy volunteers.

9 Results

10 Overall, the polymer and lipid-enriched formulation works as a film-forming product that retains

11 the therapeutic agent for long-time, ensuring its penetration and absorption through the skin, and 12 promoting skin hydration.

13 Conclusions

14 The above characteristics are useful in the clinical setting especially in the context of eczematous 15 diseases with a strong xerotic component.

17 Word count: 119 words (journal limit: 120 words)

18 Keywords: lipid-enriched vehicle; polymer; topical drug; betamethasone; spreadability; hydration 
3 The efficacy of any topical product containing an active ingredient is determined by both the

4 intrinsic activity of the active molecule and the delivery system. In order to be effective, active

5 compounds should diffuse within the formulation to the skin surface, across several tissue layers

6 (epidermis and dermis) permeating into the uppermost layer (stratum corneum) of the skin and

7 then are adsorbed into the blood capillaries and redistributed through local vasculature network. ${ }^{1}$

8 The type of vehicle influences the ability of an active agent to penetrate into the skin, and

9 therefore is associated with the potency and efficacy of topical drugs. In fact, the intrinsic activity

10 of an ingredient, or its capability to express efficacy, determines its functional profile, but does not

11 guarantee the efficacy of the final formulation. To express its function, the active ingredient must

12 be delivered to the site of action at relevant concentrations and at the right time. In addition,

13 vehicles could also directly influence the healing process, by acting in synergism with the active

14 compound to recover the altered skin physiology and alleviate dermatologic disorders. For

15 example, in the management of atopic dermatitis and psoriasis it is important to offer effective

16 treatments that relieve the dryness of the eczematous skin. Topical application of lipids can

17 change the mechanical properties of the skin by enhancing its elasticity and moisture. Since by

18 nature oils are hydrophobic, they support the lipids of the stratum corneum (SC), enhancing the

19 skin barrier functions. ${ }^{2}$ Therefore, skin occlusion by oils increases the SC water content.

20 At the same time, occlusion obtained by the formation of a lipid film on the skin surface is widely

21 utilized to enhance the penetration of topical drugs in clinical practice. In fact, in occlusion

22 condition, the SC swells for the increased water content, making lipid barrier less compact and

23 promoting the passage of nonpolar substances. ${ }^{3}$ 
1

2

3

4

5

6

7

8

9

Therefore, on the one hand occlusion reduces TEWL and increases hydration, on the other excessive occlusion can cause excessive accumulation of water in the stratum corneum and weaken the skin's barrier. Therefore, the occlusion must be chosen and balanced correctly according to the desired effect.

Moreover, vehicles should be non-irritating, non-allergenic, and cosmetically acceptable. ${ }^{4}$ In fact, cosmetic properties of topical products are should be taken into account as they affect patients' appreciation, and therefore acceptance and adherence to treatment. For instance, studies on psoriasis patients' preferences and satisfaction with treatment showed that adherence to psoriasis treatment is poor both in Europe ${ }^{5}$ and the US, ${ }^{6}$ although the great disease burden and impact on quality of life. The most important reasons for lack of adherence to topical treatments are frustration with treatment efficacy, distress associated with long-time administration, and fear of side effects. ${ }^{4}$ In this light, new designed vehicles could contribute to address some of these issues. Lotions, ointments, gels, sprays, creams and powders have been used as vehicles for topical drugs. ${ }^{7}$ In particular, ointments promote a long-lasting hydration, and are therefore indicated for dry excoriated skin. Although ointment represents the best choice for long-term penetration of active molecules and for the positive effects on skin barrier, their greasy texture reduces the cosmetic acceptance and limit usage. ${ }^{7}$ On the other hand, creams, as oil-in-water emulsions (aqueous creams) or water-in-oil emulsions (oily creams), are usually preferred because they are more readily spread and adsorbed by the skin, and are less greasy. ${ }^{7}$ A polymer-lipid-enriched cream has been recently developed ${ }^{8}$ and may represent a good compromise between efficiency as long-term delivery system, and cosmetic properties, in dry skin associated diseases. ${ }^{9}$ Since this vehicle has not been adequately investigated in the past, in the first phase of this study we compared the performances of two commercial pharmaceutical products containing betamethasone-17-valerate (hence, betamethasone) at the same concentration (0.1\%): 
betamethasone/fusidic acid cream (Fucimixbeta ${ }^{\circledR}$, Leopharma, Ballerup, Denmark. Lot:

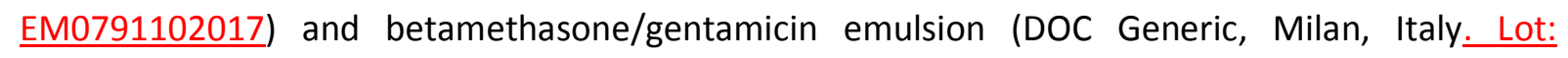
8155400). These two topical products were chosen because they -differ in the vehicle, the first being a polymer-lipid-enriched cream and the second a conventional oil in water (O/W) emulsion.

5 We decided to evaluate two commercially-available formulations known for their efficacy, with the most similar active ingredients possible, but carried in one case in a polymer and lipid-

\section{Material and methods}

EpiDerm ${ }^{T M}$ Reconstructed Human Epidermis (RHE) model and treatments

Reconstructed human epidermis (RHE) was used as a model of skin tissue. EpiDerm ${ }^{T M}$ Tissue Model has been purchased from MatTek (MatTek In Vitro Life Science Laboratories, Bratislava, Slovak Republic). Upon reception, tissues were transferred in SkinEthic ${ }^{\mathrm{TM}}$ Maintenance Medium and kept at $37{ }^{\circ} \mathrm{C}$ in a humidified $5 \% \mathrm{CO}_{2}$ atmosphere.

EpiDerm $^{\mathrm{TM}}$ Reconstructed Human Epidermis (RHE) model is a 3D epidermal tissue grown at the airliquid interface from normal human keratinocytes. The model is similar to epidermis, presenting all differentiated cellular layers, and all properties as a functional barrier.

Before treatment application, the medium was withdrawn and fresh medium was added. $10 \mu \mathrm{L}$ of the two different products (betamethasone/fusidic acid polymer lipid-enriched cream and 
1 betamethasone/gentamicin O/W emulsion) were applied over RHE tissues in a single dose for 24

2 or 48 hours. Medium aliquots were collected and centrifuged for 20 minutes to remove insoluble

3 impurity and tissue debris at $1000 \times \mathrm{g}$ at $4{ }^{\circ} \mathrm{C}$. The clear supernatants were stored at $-20^{\circ} \mathrm{C}$ until

4 analysis. After washing with PBS and snap-freezing in liquid nitrogen, the RHE tissues were

5 homogenized in a glass potter with $150 \mu \mathrm{l}$ of PBS on ice. The homogenates were then centrifuged

6 for 5 minutes at $5000 \times g$ and the supernatants were stored at $-20^{\circ} \mathrm{C}$. All procedures were repeated

7 four times for each group.

8 ELISA

9 Betamethasone levels were determined, by an ELISA kit (Elabscience Biotechnology Co., Ltd), in 10 the RHE culture media and homogenates at different times (24 or $48 \mathrm{~h}$ ). ELISA was performed

11 following the instructions accompanying the kit. Measurements were conducted with a microplate 12 reader at $450 \mathrm{~nm}$. Betamethasone was used as the standard for the calibration. All samples were 13 assayed in duplicate. The lower limit of detection for betamethasone was $0.1 \mathrm{ng} / \mathrm{mL}$. Results are 14 expressed as $\mathrm{ng} / \mathrm{mL}$.

15 Spreadability test

16 The data were obtained with a TA HDi 500 Texture Analyzer (Stable Micro System, Godalming, 17 Surrey, UK) by using the TMS Butter \& Margarine Spreadability Jig.

18 The two topical products. The spreadability test was carried out on 3 replicates performed on each 19 sample, under the following conditions:

20 - force measure in compression mode;

21 - pre-start speed $5.00 \mathrm{~mm} / \mathrm{s}$;

22 - test speed $3.00 \mathrm{~mm} / \mathrm{s}$;

23 - post-test speed $10.00 \mathrm{~mm} / \mathrm{s}$;

24 - distance $23 \mathrm{~mm}$; 
1 - load cell $5 \mathrm{~kg}$.

2 This test provided is associated with 4 parameters: firmness, work of shear, stickiness and work of

3 adhesion.

$4 \quad$ Test on human volunteers: subjects and procedures

520 healthy volunteers were recruited in this study. Subjects were properly informed about the

6 scope and potential risk (direct or secondary effects) of the investigation and also advised to

7 record any discomfort they could have been experienced. Each enrolled subject has provided a

8 written, informed consent.

9 Inclusion criteria were:

10 - caucasian origin;

11 - adults aged $18-45$ years;

12 - absence of any diseases immediately before or during the study.

13 Exclusion criteria were:

14 - subjects with in-progress topical or systemic treatment with any drug that may affect the

15 outcome of the test (anti-inflammatories, steroids, etc.);

16 - pregnancy or breastfeeding;

17 - subjects affected from skin diseases in the areas selected to receive the treatment;

18 - subjects affected from dermatitis, psoriasis or other dermatological diseases;

19 - subjects with known sensitivity to the tested product ingredients.

20 Reasons for the premature withdrawal from the study were the free choice of the enrolled

21 volunteer or the occurrence of adverse events (e.g. irritative or allergic reactions).

22 Also the pharmaceutical vehicles without active ingredients were evaluated to determine their

23 cosmetic properties. The vehicles had the following composition: 
1 - Vehicle FB01: aqua, petrolatum, paraffinum liquidum, cetearyl alcohol, steareth-21, potassium

2 sorbate, hydroxypropylmethylcellulose, methylparaben, citric acid, propylparaben.

3 - Vehicle GB01: aqua, cetearyl alcohol, paraffinum liquidum, petrolatum, ceteareth-20, 4 potassium sorbate, methyl paraben, propyl paraben.

5 The tested products were stored at room temperature and protected from light. One hundred and

6 ten $\mathrm{mg}( \pm 10 \mathrm{mg})$ of each product were applied on a predefined area of $35 \mathrm{~cm}^{2}(7 \times 5 \mathrm{~cm})$ on the

7 volar surface of the forearm, spreading approximately for 30 seconds. The products were applied

8 twice per day for 14 days. A control untreated area was identified on each volunteer's forearm

9 and the subjects were asked to avoid the application of cosmetic products to this zone.

10 Each volunteer was subjected to instrumental examinations to measure the skin barrier capacity

11 and the cutaneous hydration, at the following time period: at the beginning of the study (TO), after

127 days from the first application (T7) and at the end of the treatment (T14).

13 The following tests were performed:

14 - TEWL (TransEpidermal Water Loss): a DermaLab Combo Skinlab (Cortex - Denmark) equipped 15 with the TEWL probe was used to evaluate variations of transepidermal water loss through the 16 skin. A high level of TEWL may indicate a damage to the skin barrier which can be caused by 17 chemicals, physical insult or pathological conditions (dermatitis, eczema, psoriasis).

18 - Corneometer $^{\oplus}$ : a Derma Unit SSC 3 (Courage+Khazaka) was used to evaluate changes in 19 cutaneous hydration. The device was equipped with a specific probe for the measurement of 20 moisture (Corneometer $\left.{ }^{\circledR}\right)$. The water present in the skin causes a change of the capacitance 21 proportional to its content, giving a measure in arbitrary units of skin hydration.

22 The test took place at the following environmental conditions: temperature of $21 \pm 1^{\circ} \mathrm{C}$ and 23 humidity of $45 \pm 5 \%$. The volunteers acclimated for 15 minutes and skin was carefully cleaned 24 before measurement. 
Data were analysed by descriptive statistics. Mean values were compared applying the Student's

3 t-test for paired data. A p value $<0.05$ was considered statistically significant.

4

\section{$5 \quad$ Results}

6 Betamethasone levels

7 The ELISA assays performed on the RHE culture media of betamethasone/fusidic acid cream-

8 treated tissues and betamethasone/gentamicin O/W emulsion-treated tissues detected similar 9 concentration of betamethasone at both time-points evaluated (24 hours and 48 hours) (Table 1).

Similarly, the values of betamethasone measured in the RHE homogenates were also comparable at each time-point (Table 2). Overall, no significant differences between betamethasone/fusidic acid cream and betamethasone/gentamicin O/W emulsion were observed in terms of release of betamethasone, both in culture media and in homogenized tissues.

Spreadability Test

Table 3 reports the values of the four parameters evaluated on the samples analyzed. Firmness and work of shear are parameters indicating the energy required to deform the sample, therefore high firmness values as well as high work of shear values indicate a low spreadability and an elevated friction in the rubbing process. In this light, the two analyzed products exhibited significant differences in term of spreadability: betamethasone/fusidic acid cream obtained higher values of firmness and work of shear, resulting less spreadable compared with betamethasone/gentamicin O/W emulsion. Stickiness and work of adhesion parameters provided information regarding the cohesiveness and resistance of the sample, and are correlated with skinadhesion. The higher values obtained by betamethasone/fusidic acid cream compared with 
1 betamethasone/gentamicin $\mathrm{O} / \mathrm{W}$ emulsion indicated that former product resulted more adhesive

2 than the latter.

$3 \quad$ Transepidermal Water Loss and cutaneous hydration

419 out of 20 (95\%) enrolled subjects were females; the mean age was 25 years (range: 21-44

5 years). 19 volunteers completed the test (one person did not return for the $7^{\text {th }}$ day control).

6 Table 4 summarizes the results of the skin hydration evaluation. The application of -FL01 and GB01 vehicles significantly increased skin hydration, whereas the control values remained unchanged.

8 The increase was particularly evident for FL01 values. At T7 the skin hydration in FL01 treated area

9 was significantly higher than the GB01 value $(p<0.05)$.

10 Results obtained from the TEWL assay are summarized in Table 5. A decrease in TEWL indicates a 11 restoration of the skin barrier (reduction of transepidermal evaporation) and as known restoring

12

13

14 the skin barrier causes an increase in hydration. The changes detected in TEWL values during the study protocol were not statistically significant, both for vehicles and control. Noteworthy, the values measured in the control area were almost constant whereas, in the area treated with GB01, after an initial decrease in the first treatment period (7 days), TEWL values greatly increased after 14 days. On the other hand, in the area treated with FL01, a gradual reduction of the TEWL values during the period of the product application was observed, suggesting an improvement in skin barrier function.

\section{Discussion}

For skin diseases associated with dry skin (atopic dermatitis, psoriasis), a topical drug in a lipid vehicle could be particularly beneficial since the pharmacologic effects of the active agents is enhanced by the moisturizing properties of vehicle formulation. 
1 Vehicle formulations with high-lipid content (e.g. ointments) form an occlusive layer that gradually

2 interacts with the surface, promoting, the penetration of drugs into the skin, skin barrier

3 functions, and skin hydration.

4 However, increasing the lipid content of a cream can also have a negative impact on the release of

5 lipid-soluble active principles, such as betamethasone, due to the greater affinity of the molecule

6 for the lipid components of the cream than for the skin. In fact, it is known that ointments release

7 a smaller amount of lipid-soluble active ingredients, although for longer period, compared to

8 creams. Moreover, high-lipid content formulations are greasy and less cosmetically acceptable;

9 the poor cosmetic elegance of a topical drug can negatively impact adherence to therapy.

A polymer lipid-enriched cream could represent a suitable compromise between effective topical administration of active agents and cosmetic elegance, because of its emollient properties, with the advantage of a lower greasiness compared to ointments.

Skin penetration variability is evident for corticosteroids; the same molecule could result in variable potency in view on the vehicle used in the delivery. ${ }^{7}$

Betamethasone-17-valerate is a medium potency glucocorticoid, currently used as reference in clinical pivotal studies of new glucocorticosteroids. In this study, betamethasone release and distribution were investigated in RHE treated with two different formulations. In particular we found of interest to compare a betamethasone-based, conventional oil in water emulsion with a betamethasone-based, polymer lipid-enriched cream, that is characterized by higher lipid content and a polymer component (hydroxypropylmethylcellulose) to reduce the tendency of the lipid part of the formulation to retain the betamethasone. Although the in vitro model used to compare the betamethasone delivery over time does not exactly reflect the use of the drug, it takes into account that the products are applied by patients several times a day for several days just to have a more or less constant product persistence on the skin. In our previous studies, we have verified 
that the model used in this study is well suited for the comparison of absorption in different

2

vehicles. The two tested formulations resulted equivalent in term of release and distribution of betamethasone in culture media and cells. Moreover, the mechanical properties of these two

4 formulations, evaluated by an empirical rheological test, were different. In the spreadability test,

5 the polymer lipid-enriched cream resulted more adhesive and therefore more cohesive than O/W

6 emulsion and it works as a film forming product that retains the therapeutic agent in place for

7 longer time and in intimate contact with the surface of the skin. The enhanced properties of the

8 polymer lipid-enriched cream could be related to the polymeric film-forming system (FFS) used in

9 the cream. Cellulose derivatives, such as hydroxypropyl methylcellulose (HPMC) or hypromellose,

10 form a polymeric film that allows establishing a drug reservoir on the skin surface from which a

11 long-lasting drug release could be sustained. ${ }^{10}$

12 In fact, the persistence and resistance to removal (i.e. by washing and wear) of a topical

13 formulation, as well as a complete skin contact, are of great relevance for prolonged delivery.

14 Therefore, a successful formulation would require: high plasticity to follow the skin movements;

15 strong adhesion for, long-lasting delivery and penetration of the drug. ${ }^{10}$ Polymeric FFS has been 16 already used for the skin delivery of steroids for systemic therapies; however it could also 17 represent an attractive, alternative new delivery system for topical drugs in dermatology. ${ }^{10}$ 18 Despite the polymer lipid-enriched cream has a high content of oil apolar phase, compared with 19 O/W emulsion, the delivery of betamethasone was overall similar for the two tested products. 20 Although a larger amount of oily phase should have resulted in a greater retention of 21 betamethasone in the vehicle, by limiting its penetration through the skin, the release is still 22 guaranteed at a level comparable to that of the reference product. This effect could be explained 23 by the greater adhesive properties of betamethasone/fusidic acid polymer lipid-enriched cream 
1 that may result in prolonged substantivity. This allows the formation of a reservoir allowing

2 release of the active compound over a longer period of time. ${ }^{10}$

3 The FB01 and GB01 vehicles properties were also tested on skin of human healthy volunteers.

4 Although TEWL differences are not significant and it is not possible to draw conclusions, the

5 application of FBO1 vehicle led to a significant increase of cutaneous hydration. Since a trend to

6 TEWL values decrease for FB01 vehicle was recorded during the study, the increased skin

7 hydration could be associated with an improvement of skin barrier function. GB01 vehicle also

8 induced a small increase of skin hydration, however this result did not seem to be related to a

9 modified skin barrier action.

The higher moisturizing properties and the lower TEWL represent an advantage especially in the case of marked xerosis and barrier damage, as in the case of psoriasis and atopic dermatitis.

Overall, the above-described findings may have major clinical relevance, since a cosmetic acceptable vehicle formulation with high content of lipids can contribute, at least in part, to a longer and deeper delivery of the active compound and skin hydration, and promote adherence to dermatological treatment. ${ }^{10}$

The preliminary results, obtained both in tissues and in healthy volunteers, represent an interesting starting point in the dermatological scenario, which has topical treatments as the mainstay of many regimens. This study shows that the vehicle plays a key role in the treatment of dermatological diseases, because it guarantees and/or reduces the release of the active principles within the skin (due to its inherent characteristics), and it can also improve skin conditions and patients' compliance and adherence to treatment. However, some limitations (e.g., lack of rheological assessment) should be acknowledged.

According to the tests carried out, we can observe that the two formulation tested showed different performances with equal quantity of active principle released, and that it is reasonable to 

assume that differences may be due to the vehicle. Although these data are rather preliminary,

2 they support further investigations aimed to better clarify the mechanisms underlying the here

3 described properties of polymer lipid-enriched cream.

4 Conclusion

5 When compared with the O/W emulsion, the polymeric lipid-enriched cream showed a similar 6 release of active ingredient into the skin, a decreased spreadability, and an increased viscosity and 7 surface tension (a measure of cohesiveness). The polymeric lipid-enriched formulation, also

8 resulted more skin-adhesive, working as a film-forming product that retains the therapeutic agent

9 for long-time. It also promotes skin hydration, probably by decreasing trans-epidermal water loss

10 and improving skin barrier properties. Collectively, these properties may contribute to improve

11 adherence to treatment in dermatology.

12 The above characteristics represent an interesting synergistic approach in the clinical setting 13 especially in the context of eczematous diseases with a strong xerotic component.

\section{Acknowledgements}

We thank Luca Giacomelli, PhD, and Sara Parodi, PhD, who provided medical writing support.

\section{Funding}

This study was supported by Grant 2016 to S.M. from Consorzio Futuro in Ricerca (Ferrara, Italy).

\section{References}

[1] Rosen MR. Delivery System Handbook for Personal Care and Cosmetic Products: Technology, Applications and Formulations, 1st ed.; Elsevier Science, 2005. ISBN-13: 9780815515043. 
1 [2] Stamatas GN, de Sterke J, Hauser M, von Stetten O, van der Pol A. Lipid uptake and skin

2 3 occlusion following topical application of oils on adult and infant skin. J. Dermatol. Sci. 50, 135-142 (2008).

[3] Zhai H, Maibach HI. Occlusion vs. skin barrier function. Skin Res. Technol. 8, 1-6 (2002).

[4] Stein Gold LF. Topical Therapies for Psoriasis: Improving Management Strategies and Patient Adherence. Semin. Cutan. Med. Surg. 35, S36-44 (2016).

[5] Belinchón I, Rivera R, Blanch C, et al. Adherence, satisfaction and preferences for treatment in patients with psoriasis in the European Union: a systematic review of the literature. Patient Prefer. Adherence. 10, 2357-2367 (2016).

[6] Feldman SR. Disease burden and treatment adherence in psoriasis patients. Cutis. 92, 258263 (2013).

[7] Weiss SC. Conventional topical delivery systems. Dermatol. Ther. 24, 471-476 (2011).

[8] Girolomoni G, Mattina R, Manfredini S, et al. Fusidic acid betamethasone lipid cream. Int. J. Clin. Pract. 184, 4-13 (2016).

[9] Frederiksen K, Guy RH, Petersson K. Formulation considerations in the design of topical, polymeric film-forming systems for sustained drug delivery to the skin. Eur. J. Pharm. Biopharm. 91, 9-15 (2015).

[10] Frederiksen K, Guy RH, Petersson K. The potential of polymeric film-forming systems as sustained delivery platforms for topical drugs. Expert Opin. Drug Deliv. 13, 349-360 (2016). 
1

2

3

4

\section{5}

6

7

8

9

10

11

12

13

14

\section{Executive summary}

Background

- We compared the performances of two different commercial products both based on betamethasone and an antibiotic but using different pharmaceutical vehicles: a polymer and lipid-enriched cream and a conventional oil in water (O/W) emulsion.

Methodology

- Evaluation was conducted on a reconstructed human epidermis (RHE) model.

- Skin barrier properties and cutaneous hydration of the solely two vehicles were evaluated on

30 human healthy volunteers.

$\underline{\text { Results }}$

- Overall, the polymer and lipid-enriched formulation works as a film-forming product that retains the therapeutic agent for long-time, ensuring its penetration and absorption through the skin, and promoting skin hydration.

\section{Conclusions}

- The above characteristics are useful in the clinical setting especially in the context of eczematous diseases with a strong xerotic component. 
1

2

3

4

5

6

7

8

9

10

11

12

13

14

15

16

17

18

19

20

21

22

23

24

25

26

27

28

29

30

31

32

33

34

35

36

37

38

39

40

41

42

43

44

45

46

47

48

49

50

51

52

53

54

55

56

57

58

59

60
1

2

3

4

5 Tables

6 Table 1. Levels of betamethasone in the Reconstructed Human Epidermis (RHE) culture media

\begin{tabular}{|l|c|c|c|}
\hline \multicolumn{1}{|c|}{ ng/mL } & Time-points & Mean & SD \\
\hline Betamethasone/fusidic acid cream & $24 \mathrm{~h}$ & 6.3 & 0.4 \\
\hline Betamethasone/gentamicin O/W emulsion & $24 \mathrm{~h}$ & 6.8 & 0.5 \\
\hline Betamethasone/fusidic acid cream & $48 \mathrm{~h}$ & 7.1 & 0.3 \\
\hline Betamethasone/gentamicin O/W emulsion & $48 \mathrm{~h}$ & 6.9 & 0.3 \\
\hline
\end{tabular}

7 SD: standard deviation

8

9

10

11

12

13

14

15

16

17

18

19

20

21

22

23

24 
1

2

3

4

5

6 Table 2. Levels of betamethasone in the Reconstructed Human Epidermis (RHE) homogenates

\begin{tabular}{|l|c|c|c|}
\hline \multicolumn{1}{|c|}{$\mathbf{n g} / \mathrm{mL}$} & Time-points & Mean & SD \\
\hline Betamethasone/fusidic acid cream & $24 \mathrm{~h}$ & 3.4 & 1.1 \\
\hline Betamethasone/gentamicin O/W emulsion & $24 \mathrm{~h}$ & 5.2 & 0.3 \\
\hline Betamethasone/fusidic acid cream & $48 \mathrm{~h}$ & 3.7 & 0.6 \\
\hline Betamethasone/gentamicin O/W emulsion & $48 \mathrm{~h}$ & 3.1 & 0.3 \\
\hline
\end{tabular}

7 SD: standard deviation

8

9

10

11

12

13

14

15

16

17

18

19

20

21

22

23

24

25

26

59

60

https://mc04.manuscriptcentral.com/fs-tde 
1

2

3

4

5

6

7

8

9

10

11

12

13

14

15

16

17

18

19

20

21

22

23

24

25

26

27

28

29

30

31

32

33

34

35

36

37

38

39

40

41

42

43

44

45

46

47

48

49

50

51

52

53

54

55

56

57

58

59

60
1

2

3

4

5

6 Table 3. Outcomes of the performed spradability test. Data are expressed as mean \pm standard

7 deviation.

\begin{tabular}{|l|c|c|c|c|}
\hline Sample & $\begin{array}{c}\text { Firmness } \\
\mathbf{( g )}\end{array}$ & $\begin{array}{c}\text { Work of } \\
\text { shear } \\
\left(\mathbf{g}^{*} \mathbf{s}\right)\end{array}$ & $\begin{array}{c}\text { Stickiness } \\
\mathbf{( g )}\end{array}$ & $\begin{array}{c}\text { Work of } \\
\text { adhesion } \\
(\mathbf{g *})\end{array}$ \\
\hline Betamethasone/fusidic acid cream & $527 \pm 28$ & $414 \pm 18$ & $554 \pm 25$ & $124 \pm 3$ \\
\hline $\begin{array}{l}\text { Betamethasone/gentamicin O/W } \\
\text { emulsion }\end{array}$ & $164 \pm 5$ & $127 \pm 4$ & $171 \pm 2$ & $44 \pm 2$ \\
\hline
\end{tabular}

8

9

10

11

12

13

14

15

16

17

18

19

20

21

22

23

24

25

26 
1

2

3

4

5

6

7

\section{8} 9

10

\begin{tabular}{|l|c|c|c|c|c|c|}
\hline Time-points & \multicolumn{3}{|c|}{ Cutaneous Hydration } & \multicolumn{3}{c|}{ \% Variation } \\
\hline & Control & FB01 & GB01 & Control & FB01 & GB01 \\
\hline T0 & $32.6 \pm 3.0$ & $36.1 \pm 2.8$ & $37.2 \pm 3.4$ & - & - & - \\
\hline T7 & $34.8 \pm 2.5$ & $45.9 \pm 2.7$ & $42.9 \pm 2.8$ & 6.7 & 27.1 & 15.3 \\
\hline T14 & $31.0 \pm 1.5$ & $43.9 \pm 2.7$ & $42.2 \pm 2.2$ & -4.9 & 21.6 & 13.4 \\
\hline
\end{tabular}

11

12

13

14

15

16

17

18

19

20

21

22

23

24

25

26

27

Table 4. Assessment of the cutaneous hydration at different timepoints. Data are expressed in arbitrary units as mean and as $\%$ variation from T0 considering all participants ( 5 measurements/participant). T0: beginning of the study; T7: 7 days from the first application; T14: end of the treatment.

(1)

3

4

5

6

17

8

19
20
21
22
23
24
25
26
27


5 Table 5. Assessment of the transepideral water loss at different timepoints. Data are expressed as 6 mean and as \% variation from T0 considering all each participants ( 3 measurements/participant). 7

\begin{tabular}{|l|c|c|c|c|c|c|}
\hline Time-points & \multicolumn{3}{|c|}{ TEWL (g/m $\left.\mathbf{m}^{\mathbf{h}}\right)$} & \multicolumn{3}{c|}{ \% Variation } \\
\hline & Control & FB01 & GB01 & Control & FB01 & GB01 \\
\hline T0 & $2.1 \pm 0.4$ & $2.6 \pm 0.6$ & $2.8 \pm 0.7$ & - & - & - \\
\hline T7 & $2.0 \pm 0.6$ & $2.2 \pm 0.6$ & $2.4 \pm 0.6$ & -4.8 & -15.4 & -14.3 \\
\hline T14 & $1.9 \pm 0.4$ & $2.0 \pm 0.3$ & $3.1 \pm 0.7$ & -9.5 & -23.1 & 10.7 \\
\hline
\end{tabular}

8

9

10 